\title{
On Cartesian Products which Determine Few Distinct Distances
}

\author{
Cosmin Pohoata \\ Department of Mathematics \\ California Institute of Technology \\ Pasadena, U.S.A. \\ apohoata@caltech.edu
}

Submitted: Mar 21, 2018; Accepted: Nov 18, 2018; Published: Jan 11, 2019

(C) The author. Released under the CC BY-ND license (International 4.0).

\begin{abstract}
Every set of points $\mathcal{P}$ determines $\Omega(|\mathcal{P}| / \log |\mathcal{P}|)$ distances. A close version of this was initially conjectured by Erdös in 1946 and rather recently proved by Guth and Katz. We show that when near this lower bound, a point set $\mathcal{P}$ of the form $A \times A$ must satisfy $|A-A| \ll|A|^{2-\frac{2}{7}} \log ^{\frac{1}{7}}|A|$. This improves recent results of Hanson and Roche-Newton.
\end{abstract}

Mathematics Subject Classifications: 05D99

\section{Introduction}

Let $\mathcal{P}$ be a set of points in plane, and let $\Delta(\mathcal{P})$ denote the set of squares of distances spanned by $\mathcal{P}$. In other words,

$$
\Delta(\mathcal{P})=\left\{\left(p_{1}-q_{1}\right)^{2}+\left(p_{2}-q_{2}\right)^{2}:\left(p_{1}, p_{2}\right),\left(q_{1}, q_{2}\right) \in \mathcal{P}\right\} .
$$

In [7], Guth and Katz showed that $\Delta(\mathcal{P}) \gg|\mathcal{P}| / \log |\mathcal{P}|$, where $\gg$ represents the usual Vinogradov symbol. When $P=A \times B$ for some finite sets of reals $A$ and $B, \Delta(A \times B)=$ $(A-B)^{2}+(A-B)^{2}$, so this says that

$$
\left|(A-B)^{2}+(A-B)^{2}\right| \gg \frac{|A||B|}{\log |A||B|} .
$$

In [5], Erdös originally conjectured that all sets $\mathcal{P}$ should determine $\Omega(|\mathcal{P}| / \sqrt{\log |\mathcal{P}|})$ distinct distances, so the Guth-Katz bound is almost optimal. Nonetheless, very little is known for sets that achieve this bound. It is widely believed that sets with $O(|\mathcal{P}| / \log |\mathcal{P}|)$ distinct distances should come from some type of lattice. This is very well-motivated by the following beautiful result of Bernays [2], which generalizes a classical theorem of Landau. 
Theorem 1. Let $f(x, y)=a x^{2}+b x y+c y^{2}$ for integers $a, b, c \in \mathbb{Z}$, such that the determinant $b^{2}-4 a c$ is not an integer square. Then, the number of integers between 1 and $n$ that can be expressed as $f(u, v)$ with $u, v \in \mathbb{Z}$ is $O(n / \sqrt{\log n})$.

Using Theorem 1, one can easily check that sets with $O(n / \sqrt{\log n})$ distinct distances are given by $\sqrt{n} \times \sqrt{n}$ subsets of the integer lattice, the (equilateral) triangular lattice, or, more exotically, by the rectangular lattice

$$
\mathcal{L}_{r}=\{(i, j \sqrt{r}) \mid i, j \in \mathbb{Z}, 1 \leqslant i, j \leqslant n\},
$$

for every integer $r>1$. We refer the reader to [14] for a more detailed presentation of this discussion, where Sheffer also points out that unlike the first two examples, the latices $\mathcal{L}_{r}$ do not span squares or equilateral triangles.

In this paper, we will only take a look at sets that come from cartesian products, and show that whenever they determine few distinct distances they must exhibit some additive structure. Specifically, when $\mathcal{P}=A \times A$, we show that when the Guth-Katz bound is close to being tight, we have that

$$
|A-A| \ll|A|^{2-\frac{2}{7}} \log ^{\frac{1}{7}}|A| .
$$

In light of the bipartite distance problems discussed by Brunner and Sharir in [3] and by Sheffer and the author in [2], we also consider the problem of showing that if there are few distinct distances between two cartesian products $A \times A$ and $B \times B$, then one of $A$ or $B$ has additive structure. We state both of these results more formally below.

Theorem 2. Suppose $A$ is a finite set of real numbers and let $\Delta(A \times A)$ be the set of distances spanned by $A \times A$. Then,

$$
|A-A| \ll|\Delta(A \times A)|^{\frac{6}{7}} \log ^{\frac{1}{7}}|A|,
$$

or equivalently $|D| \ll\left|D^{2}+D^{2}\right|^{\frac{6}{7}} \log ^{\frac{1}{7}}|D|$, where $D$ denotes the difference set $A-A$.

Theorem 3. Suppose $A$ and $B$ are finite sets of real numbers and let $\Delta(A \times A, B \times B)$ be the set of distances between points in $A \times A$ and points in $B \times B$. Then,

$$
\min \{|A-A|,|B-B|,|A-B|\} \ll|\Delta(A \times A, B \times B)|^{1-\frac{13}{205}} \cdot E(A, B),
$$

where

$$
E(A, B)=\min \left\{\log \frac{3}{205}|A|, \log \frac{3}{205}|B|\right\}
$$

In particular, if $|\Delta(A \times A)| \ll|A|^{2}$ holds in Theorem 2, then $|A-A| \ll|A|^{2-\frac{2}{7}} \log ^{\frac{1}{7}}|A|$. This improves a recent theorem by Hanson [8], who showed that under this hypothesis we have that $|A-A| \ll|A|^{2-\frac{1}{8}}$. In the meantime this was also sharpened by Roche-Newton in [13], who showed $|A-A| \ll|A|^{2-\frac{2}{11}}$, but the estimate from Theorem 2 is stronger. Our proof will rely on the sum-product estimate of Solymosi from [16] as a black-box: 
Theorem 4. Let $S \subset \mathbb{R}$ be a set. Then

$$
|S+S|^{2}|S S| \geqslant \frac{|S|^{4}}{4\lceil\log |S|\rceil} .
$$

The proof of Theorem 3 will rely on two results. The first one is the following Lemma by Balog [1], which comes from Solymosi's original idea for Theorem 4.

Lemma 5. Let $R, S, T$ be finite sets of real numbers. Then

$$
|R T+R T||S T+S T| \gg|R / S||T|^{2} .
$$

The second one is the following Lemma due to Shkredov, which is Theorem 3 in [15] (and the statement of which should be in some sense compared to that of Theorem 2 above).

Lemma 6. Let $A \subset \mathbb{R}$ be a finite set and let $D=A-A$. Then

$$
|D / D| \gg|D|^{1+\frac{1}{12}} \log ^{-1 / 4}|D|
$$

Last but not least, we will also need the classical Plünnecke-Ruzsa inequality, for which a simple proof can be found in [11].

Lemma 7. Let $A \subset \mathbb{R}$ be a finite set. Then

$$
|k A-\ell A| \leqslant \frac{|A+A|^{k+\ell}}{|A|^{k+\ell-1}} .
$$

\section{Proof of Theorem 2}

If $D=A-A$, then $|\Delta(A \times A)|=\left|D^{2}+D^{2}\right|$, where $D^{2}=\left\{(x-y)^{2}: x, y \in A\right\}$. We claim that

$$
|D| \ll\left|D^{2}+D^{2}\right|^{\frac{6}{7}} \log ^{\frac{1}{7}}|D| .
$$

We apply Theorem 4 for the set $S:=D^{2}$. Using the observation that $\left|D^{2} D^{2}\right|$ is equal to $|D D|$ (up to a small constant), this yields

$$
\left|D^{2}+D^{2}\right|^{2}|D D| \geqslant\left|D^{2}+D^{2}\right|^{2}\left|D^{2} D^{2}\right| \geqslant \frac{\left|D^{2}\right|^{4}}{4\left\lceil\log \left|D^{2}\right|\right\rceil} \gg \frac{|D|^{4}}{\log |D|} .
$$

On the other hand for every four real numbers $a_{1}, a_{2}, b_{1}, b_{2}$, we have that

$$
\left(b_{1}-a_{1}\right)^{2}+\left(b_{2}-a_{2}\right)^{2}-\left(b_{1}-a_{2}\right)^{2}-\left(b_{2}-a_{1}\right)^{2}=2\left(a_{2}-a_{1}\right)\left(b_{1}-b_{2}\right),
$$

which yields the inclusion

$$
2 \cdot D D \subset 2 D^{2}-2 D^{2}
$$


We emphasize here that for $X \subset \mathbb{R}$ and $c \in \mathbb{Z}_{>0}$, the set $c \cdot X$ denotes the set of scalar multiples $\{c x: x \in X\}$, whereas $c X$ denotes the sumset $\sum_{i=1}^{c} X$. The inclusion together with Lemma 7 then yield

$$
\begin{aligned}
\left|D^{2}+D^{2}\right|^{2}|D D| & =\left|D^{2}+D^{2}\right|^{2}|2 \cdot D D| \\
& \leqslant\left|D^{2}+D^{2}\right|^{2}\left|2 D^{2}-2 D^{2}\right| \\
& \ll\left|D^{2}+D^{2}\right|^{2}\left(\frac{\left|D^{2}+D^{2}\right|^{4}}{|D|^{3}}\right) .
\end{aligned}
$$

Putting the two bounds together, we conclude that

$$
\frac{\left|D^{2}+D^{2}\right|^{6}}{|D|^{3}} \gg \frac{|D|^{4}}{\log |D|}
$$

which yields

$$
|D| \ll\left|D^{2}+D^{2}\right|^{\frac{6}{7}} \log ^{\frac{1}{7}}|D|
$$

\section{Proof of Theorem 3}

For convenience, write again that $|\Delta(A \times A, B \times B)|=\left|(A-B)^{2}+(A-B)^{2}\right|$. Since

$$
\left(b_{1}-a_{1}\right)^{2}+\left(b_{2}-a_{2}\right)^{2}-\left(b_{1}-a_{2}\right)^{2}-\left(b_{2}-a_{1}\right)^{2}=2\left(a_{2}-a_{1}\right)\left(b_{1}-b_{2}\right)
$$

holds for every $a_{1}, a_{2} \in A, b_{1}, b_{2} \in B$, we have the inclusion

$$
2 \cdot(A-A)(B-B)+2 \cdot(A-A)(B-B) \subset 4(A-B)^{2}-4(A-B)^{2} .
$$

On one hand Lemma 7 gives

$$
\begin{aligned}
\left|4(A-B)^{2}-4(A-B)^{2}\right| & \leqslant \frac{\left|(A-B)^{2}+(A-B)^{2}\right|^{8}}{\left|(A-B)^{2}\right|^{7}} \\
& =\frac{\left|(A-B)^{2}+(A-B)^{2}\right|^{8}}{|A-B|^{7}} .
\end{aligned}
$$

On the other hand, the above inclusion gives

$$
\begin{aligned}
\left|4(A-B)^{2}-4(A-B)^{2}\right|^{2} & \geqslant|2 \cdot(A-A)(B-B)+2 \cdot(A-A)(B-B)|^{2} \\
& =|(A-A)(B-B)+(A-A)(B-B)|^{2} .
\end{aligned}
$$

Furthermore, Lemma 5 applied for $R=S=A-A, T=B-B$ tells us that

$$
|(A-A)(B-B)+(A-A)(B-B)|^{2} \gg\left|\frac{A-A}{A-A}\right||B-B|^{2} .
$$


By Lemma 6,

$$
\left|\frac{A-A}{A-A}\right| \gg|A-A|^{1+\frac{1}{12}} \log ^{-1 / 4}|A-A|,
$$

SO

$$
\left|4(A-B)^{2}-4(A-B)^{2}\right|^{2} \gg|A-A|^{1+\frac{1}{12}}|B-B|^{2} \log ^{-1 / 4}|A-A| .
$$

We conclude that

$$
\frac{\left|(A-B)^{2}+(A-B)^{2}\right|^{16}}{|A-B|^{14}} \gg|A-A|^{1+\frac{1}{12}}|B-B|^{2} \log ^{-1 / 4}|A-A| .
$$

By using Lemma 6 for $R=S=B-B$ and $T=A-A$ instead, we can similarly get

$$
\frac{\left|(A-B)^{2}+(A-B)^{2}\right|^{16}}{|A-B|^{14}} \gg|A-A|^{2}|B-B|^{1+\frac{1}{12}} \log ^{-1 / 4}|B-B| .
$$

Putting everything together, we get

$$
\min \{|A-A|,|B-B|,|A-B|\} \ll\left|(A-B)^{2}+(A-B)^{2}\right|^{1-\frac{13}{205}} \cdot \mathrm{E}(A, B),
$$

where

$$
\mathrm{E}(A, B)=\min \left\{\log \frac{3}{205}|A|, \log \frac{3}{205}|B|\right\} .
$$

This completes the proof.

\section{Concluding Remarks}

Theorem 2 is still far from being optimal. We conjecture that when $|\Delta(A \times A)|=o\left(|A|^{2}\right)$,

$$
|A-A| \ll|A|^{1+\epsilon}
$$

for any $\epsilon>0$. The $\epsilon$ in the conjecture is justfied by the following remark. Translate the set $A$ so that it contains 0 so that now $\left|(A-A)^{2}+(A-A)^{2}\right|=o\left(|A|^{2}\right)$ implies $\left|A^{2}+A^{2}\right|=o\left(|A|^{2}\right)$. On the other hand, using an argument similar to the one of Elekes and Ruzsa from [4], one can show that for every $A, B \subset \mathbb{R}$ we have that

$$
\left|A^{2}+B^{2}\right||A-A+B|^{2}|A-A-B|^{2} \gg|A|^{4}|B|^{2} .
$$

In particular, whenever $A=B$ and $|A-A| \ll A$, Lemma 7 yields $\left|A^{2}+A^{2}\right| \gg|A|^{2}$, so we get a contradiction.

It is worth mentioning that even assuming the full-strength of the Erdős-Szemerédi conjecture [6], which says that for any $\epsilon>0$ one has

$$
\max \left|D^{2}+D^{2}\right|,\left|D^{2} D^{2}\right| \gg|D|^{2-\epsilon^{\prime}}
$$

our proof for Theorem 2 only gives

$$
|A-A| \ll|A|^{2-\frac{4}{7}+\epsilon} .
$$


Using the updates building on the Konyagin and Shkredov improvements of Solymosi's bound $[9,10]$, one can perhaps bring

$$
|A-A| \ll|A|^{2-\frac{2}{7}} \log ^{\frac{1}{7}}|A|
$$

down to

$$
|A-A| \ll|A|^{2-\frac{2}{7}-c} \log ^{\frac{1}{7}}|A|
$$

for some small constant $c>0$, but significant improvements to Theorem 2 should perhaps first come from replacing the inequality $|D D| \leqslant\left|2 D^{2}-2 D^{2}\right|$ coming from $2 \cdot D D \subset$ $2 D^{2}-2 D^{2}$ with a more efficient argument. We believe that the Erdös-Szemerédi conjecture should imply the claim that cartesian products with $o\left(|A|^{2}\right)$ distinct distances satisfy $|A-A| \ll|A|^{1+\epsilon}$.

\section{Acknowledgments}

I would like to thank Oliver Roche-Newton, Misha Rudnev and Adam Sheffer for helpful conversations.

\section{References}

[1] A. Balog, A note on sum-product estimates, Publ. Math. Debrecen. 79 (2011), 3-4, 283-289.

[2] P. Bernays, Über die Darstellung von positiven, ganzen Zahlen durch die primitiven, binären quadratischen Formen einer nicht-quadratischen Diskriminante Ph.D Thesis (1912).

[3] A. Bruner and M. Sharir, Distinct distances between a collinear set and an arbitrary set of points, Discrete Math., to appear.

[4] G. Elekes and I. Z. Ruzsa, Few sums, many products, Studia Scientiarum Mathematicarum Hungarica 40 (2003), 301-308.

[5] P. Erdős, On some metric and combinatorial geometric problems, Discrete Math. 60 (1986), 147-153.

[6] P. Erdős, E. Szemerédi, On sums and products of integers, Studies in pure mathematics, 213-218, Birkhäuser, Basel, 1983.

[7] L. Guth and N. H. Katz, On the Erdős distinct distances problem in the plane, Ann. of Math. 2181 (2015), 155-190.

[8] B. Hanson, The additive structure of cartesian products spanning few distinct distances, Combinatorica (2017), 1-6.

[9] S. Konyagin and I. D. Shkredov, On sum sets of sets, having small product set, Proc. of the Steklov Institute of Mathematics, 290 (2015), 288-299.

[10] S. Konyagin and I. D. Shkredov, New results on sum-products in $\mathbb{R}$, Transactions of Steklov Mathematical Institute, to appear; preprint: arXiv:1602.03473. 
[11] G. Petridis, New proofs of Plünnecke-type estimates for product sets in groups, Combinatorica 32 (2012), no. 6, 721-733.

[12] C. Pohoata, A. Sheffer, Higher Distance Energies and Expanders with Structure, arXiv: 1709.06696.

[13] O. Roche-Newton, On sets with few distinct distances, arXiv:1608.02775.

[14] A. Sheffer, Point sets with few distinct distances, blog post: https://adamsheffer. wordpress.com/2014/07/16/point-sets-with-few-distinct-distances/.

[15] I. D. Shkredov, Difference sets are not multiplicatively closed, Discrete Analysis 2016: 17, 20pp.

[16] J. Solymosi, Bounding multiplicative energy by the sumset, Advances in Mathematics, Volume 222, 2 (2009), 402-408.

[17] P. Ungar, $2 N$ noncollinear points determine at least $2 N$ directions, J. Combinatorial Theory, Ser. A 33 (1982), 343-347. 